\title{
SECONDARY EMISSION MAGNETRON INJECTION GUN IN LONG PULSE MODE
}

\author{
S. A. Cherenshchikov, G.M. Ivanov, L.A. Makhnenko, KIPT, Kharkov, Ukraine.
}

\section{Abstract}

The Secondary Emission Magnetron Injection Gun (SEMIG) is relatively a new cold-cathode electron gun [1]. The advantages of the SEMIG in long pulse operations are more obvious when we apply it for power radio-frequency sources and electron linacs. Experimental facility and a method of emission excitation in the SEMIG are described. The method consist of the secondary emission multiplication on the trailing edge of the voltage pulse. The SEMIG is operated with the extern pulsedriver. Similar method is known for magnetrons. The electron beam with pulse duration up to $3000 \mu$ s and repetition rate up to $50 \mathrm{~Hz}$ (duty factor up to $15 \%$ ) is obtained.

\section{INTRODUCTION}

The main direction of accelerator development is increasing its lifetime and efficiency. The new cold cathode electron gun gives us this possibility. The gun is known as the Secondary Emission Magnetron Injection Gun (SEMIG) [1]. It is based on a new principle: selfsupported secondary emission in crossed fields. The gun may be used as injector in electron linacs [2] and radiofrequency power sources [3-6] for accelerators.

The field of gun application may be large if it could produce high current relatively long pulses of electron beam at high current. Conveniently, all high current linacs and power sources use solenoid as magnetic field sources. If the duty factor of device increases then full efficiency increases too, because solenoid feed power is constant. This shows the great importance of increasing pulse length because duty factor is in proportional of pulse length. The possibility of obtaining a long pulse in cold cathode magnetron was shown earlier [7]. Now we describe the long pulse mode in SEMIG.

\section{THE PULSE DURATION LIMITATIONS}

The limit of the current density $J$ depends on pulse length $\tau$ and cathode material. The estimation is made on the base of Kovalenko's understanding [8] of the physical processes in vacuum tube. The current density $J$ is estimated as:

$$
J=\frac{\text { const }}{\sqrt{\tau}}
$$

where for copper: const $=260\left[\mathrm{~A}^{\cdot} \mathrm{cm}^{-2 .}(\mu \mathrm{s})^{1 / 2}\right]$; for tungsten: const $=860\left[\mathrm{~A}^{\cdot} \mathrm{cm}^{-2} \cdot(\mu \mathrm{s})^{1 / 2}\right]$.

Maximum current $I$ may be estimate as

$$
I=J L \pi d
$$

where $L$ - cathode length, $d$ - cathode diameter. In our data $I$ is equal to $45 \mathrm{~A}$. The current is more large then one from conventional thermoionic cathode for the accelerator [8].

\section{THE CONDITION OF SECONDARY EMISSION EXCITATION}

The SEMIG consists of a hollow tube anode and coaxial finger-like secondary emission cathode inside it [1]. Correspondence between value of magnetic field and apply voltage pulse is chosen in order to make electrons to return the cathode (Hell's cut off relation). The start of electron multiplication occurs at the fall of the negative high voltage pulse applied to the cathode $[10,11]$. After initiation the electron emission continues at the top of the following part of the pulse [12].

The cause of secondary emission is additional energy of returned electrons from the turbulent electron flow in cross-field gap due to increasing electron space charge. Near the cathode edge the electron space charge gives axial component of the electric field. The axial component is the cause of electron injection and produces the annual electron beam.

For emission excitation the amplitude of voltage pulse and the magnetic induction must be large enough. Figures 1,2 show theory [10] and experimental boundaries [12] of excitation region in coordinates "voltageinduction".

\section{EXPERIMENTAL FACILITY}

The auxiliary firing pulse amplitude for puremetallic cathodes is high enough even when the magnetic field is optimal [12 ]. The attempts to start the secondary emission with the help of the existing submodulator was made to do it by the in previous tests and experiments were unsuccessful. That's why the decision was made to do it by the main modulator pulse which in our previous experiments ensured firing with the sufficiently high voltage decrease. The submodulator forming circuits and its pulse transformer didn't ensure producing of pulses with the microsecond range. So, the scheme of experiment was chosen as shown in Fig. 3. By this scheme the pulse from main pulse modulator 1 was used as exciting one. Position 2 is the matched cable, and position 3 is the pulse step-up transformer, put in tank 4 filled with oil. The 
pulse was fed to vacuum volume through high-voltage insulator 5, and reached the cathode 6 . The cathode 6 was situated coaxially inside the anode 7 which simultaneously worked as passing pipe. The isotropic magnetic field along the axis of cathode 6 and anode 7 was produced by solenoid 8 . The magnetic field induction could be tuned in the interval $0-0.3 \mathrm{~T}$ by changing the current in the solenoid 8 . The beam formed by the gun 6 , 7 was falling on the collector 9 . The beam current value and the pulse duration were obtained from oscillograph 10 of type C4-74, using the synchronization system 11 of Universal Injecting Complex (UIC) [9]. The long beam current pulse was formed by accumulating condenser 12 . To avoid the rectifier diodes damage in the case of gun spark-over the resistor 13 with low resistance was included in series into the gun circuit. The choke 15 was the short circuit defense of the gun. The voltage of the accumulating condenser 12 was tuned by autotransformer in limits of $0-20 \mathrm{kV}$.

\section{RESULTS}

After the pulse from modulator 1 is guided to the gun cathode 6 , relatively the grounded passage tube 7 at the fall of the pulse begins the secondary emission multiplication [10,11]. If the multiplication is large enough at the end of the pulse, self-supporting secondary emission mode is established. After the pulse ends generation of the beam is continuous due to the discharge of accumulating capacity 12. If the pulse duration increases the beam current decreases. Experiments have shown that it is possible to increase the duration of the pulse to $3,000 \mu \mathrm{s}$ at the magnetic field of $0.13 \mathrm{~T}$ and the value of capacity 12 is $0.1 \mu \mathrm{F}$.

All modes are shown in the table

$\begin{array}{llll}\begin{array}{l}\text { Number } \\ \text { the mode }\end{array} & \begin{array}{l}\text { of } \\ \text { field, T }\end{array} & \begin{array}{l}\text { Pulse } \\ \text { duration, } \mu \mathrm{s}\end{array} & \begin{array}{l}\text { Beam } \\ \text { current, A }\end{array} \\ 1 & 0.130 & 200 & 0.05 \\ 2 & 0.154 & 400 & 0.10 \\ 3 & 0.138 & 3,000 & 0.005\end{array}$

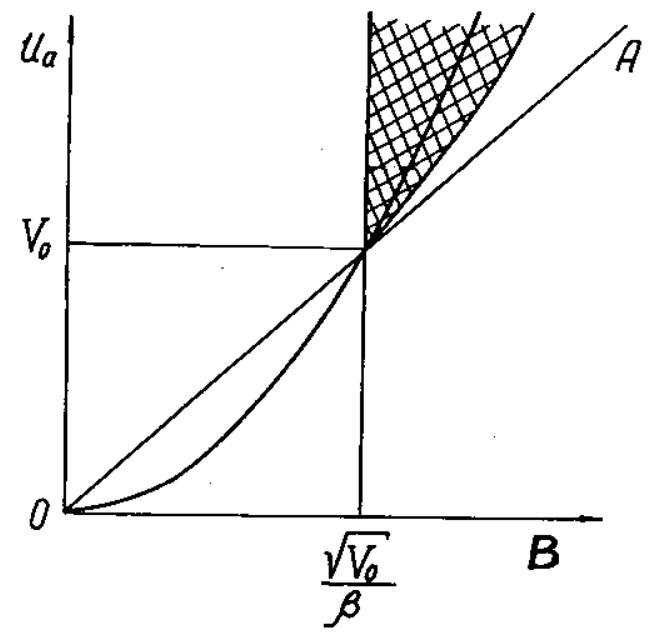

Fig.1The theory region of secondary emission cross-field excitation [10] (xxx)

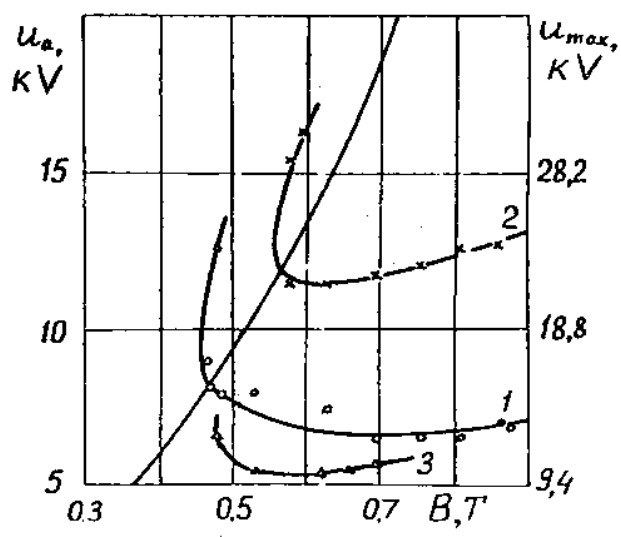

Fig.2 The experimental excitation boundaries in the magnetron [12].

At the left the pulse modulator voltage is shown. At the right the amplitude exciting pulse is shown.

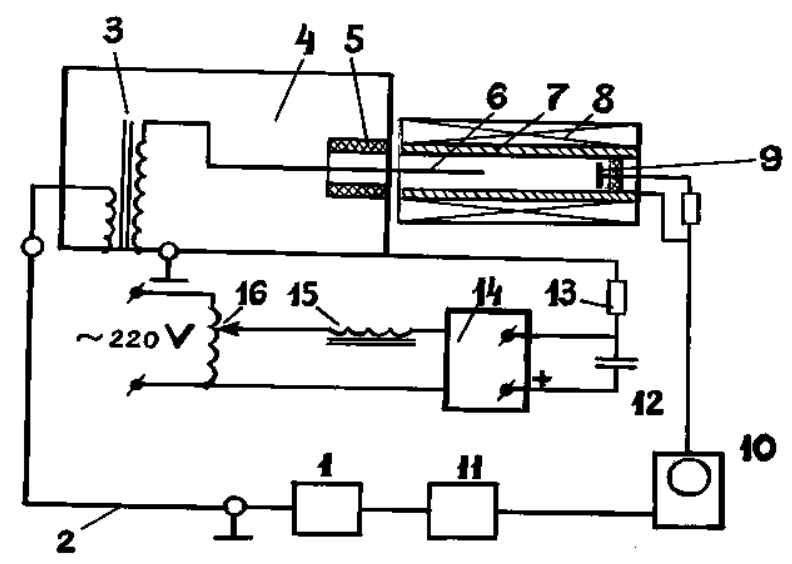

Fig.3 The shame of experimental facility:

1- main pulse modulator; 2-coacsial cable; 3- pulse transformer; 4- oil tank; 5- main insulator; 6- secondary emission cold cathode; 7- anode- (drift-tube); 8- solenoid; 9- collector (Faraday cup); 11- oscillograph; 12accumulating condenser; 13- defense resistor; 14-gun power souse (submodulator); 15- defense choke. 


\section{ACKNOWLEDGEMENT}

The author expresses his gratitude to Dr.A.Opanasenko for useful discussion of the obtained results and his assistance in the preparation of these reports.

\section{REFERENCES}

[1] ] S.A. Cherenshchikov.et al. // AIP Conference Proceeding. 1994. Vol. 337. P. 350-359.

[2] S.A.Cherenshchikov, G.M.Ivanov, L.A.Makhnenko, A.N.Opanasenko. Secondary Emission Magnetron Ingection Gun as Injector for Linac. (Poster report WEA-38 of this conferense)

[3] S.A.Cherenshchikov, A.N.Opanasenko, "Secondary Emission Magnetron Injection Gun for RF-Sources", // Abstract for V European Particle Accelerator Conference (Barselona, 10-14 June 1996).

[4] S.A.Cherenshchikov, A.N.Opanasenko, "Concept of Employing SEMIG in RF-Sources for Future Linear Colliders", // Abstract for V European Particle Accelerator Conference (Barcelona, 10-14 June 1996).

[5] S.A.Cherenshchikov A.N.Opanasenko. Prospects of Increasing Life Time and Efficiently of New Generation RF Sources by Path of Application Secondary-Emission Unheated Metallic Cathodes, Abstract for XVII Particle Accelerator Conference PAC'97, Vancouver, Canada.

[6] S.A.Cherenshchikov, G.M.Ivanov, L.A.Makhnenko,

A.N.Opanasenko, "About Possibility of Thermoionic Gun Substitution at Power Klystron Amplifier for Secondary Emission Magnetron Guns"// Voprosy Atomnoy Nauki i Tekhniki. Seriya: Yadernofiz. Issled. (Kharkov) 4,5 (31,32), pp. 40-42, 1997.

[7] S.A.Cherenshchikov, V.D.Naumenko,. "Investigation of the Magnetron Start with a Cold Secondary-Emission Cathode at the Trailing Edge of the Voltage Pulse",

Izvestija Vuzov "Radiofizika", vol. 27, pp. 250-256, February, 1984.

[8] V.F. Kovalenko. Thermal Phisic Prossesis and and Electron Vacuum Devisis. Sov. Radio. Moskow. 1975.

[9] S.A.Cherenshchikov, G.M.Ivanov, V.I.Kurilko, L.A.Makhnenko, A.N.Opanasenko, P.M.Ryabka, and, "Experimental Investigations of Electrodynamics Characteristics of Accelerating Structure STRUM-90". Journal of Technical Physics, Vol. 64, No. 4, 1994, (p.115-123 )

[10] S.A. Cherenshchikov. "About the Magnetron Start with a Cold Cathode at the Trailing Edge of the Voltage Pulse", Elektronnaja Tehnika -Ser. Electronika SVCh, ${ }^{1}$ 6, pp. 20-28, June, 1973

[11] S.A.Cherenshchikov. "Secondary-Emission Multiplication at Magnetically Insulated Discharge in a Variable Field", Izvestija Vuzov "Radiofizika", vol.27, pp.1331-1337, October, 1984.

[12] S.A.Cherenshchikov, V.D.Naumenko,. "Investigation of the Magnetron Start with a Cold Secondary-Emission Cathode on the Trailing Edge of the Voltage Pulse", Izvestija Vuzov "Radiofizika", vol. 27, pp. 250-256, February, 1984. 